Esta revista forma parte del acervo de la Biblioteca Jurídica Virtual del Instituto de Investigaciones Jurídicas de la UNAM

\title{
De la ley a la plaza: derecho, representación, participación y plena ciudadanía*
}

\author{
From the Law Books to the Public Place: Law, \\ Representation, Participation and Full Citizenship
}

Salvador O. Nava Gomar**

\section{Sumario:}

I. A manera de introducción

II. Participación ciudadana, representación y representatividad

III. Sistemas electorales

IV. El clientelismo o la instrumentalización de la participación política

V. Comentario final

VI. Bibliografía

* El texto corresponde a la comunicación presentada en el XI Congreso Iberoamericano de Derecho Constitucional "Jorge Carpizo", celebrado en Tucumán, Argentina, del 17 al 19 de septiembre de 2013.

** Magistrado de la Sala Superior del Tribunal Electoral del Poder Judicial de la Federación. El autor agradece a Mauricio del Toro y Javier Ortiz, secretarios adscritos a su ponencia por el apoyo en la elaboración del presente documento.

Recibido: 26 de mayo de 2013 Aceptado: 28 de octubre de 2013

D. R. @ 2014. Universidad Nacional Autónoma de México-Instituto de Investigaciones Jurídicas. Revista Mexicana de Derecho Electoral, núm. 5, enero-junio de 2014, pp. 87-103, 
Esta revista forma parte del acervo de la Biblioteca Jurídica Virtual del Instituto de Investigaciones Jurídicas de la UNAM

\section{Resumen:}

El presente documento es una reflexión general sobre algunos aspectos centrales del debate actual sobre la participación política en las sociedades complejas, a partir de identificar aquellas prácticas políticas que inciden directamente en la representación y en la representatividad de los sistemas electorales, en particular, los efectos nocivos del clientelismo y el patronazgo, ante la necesidad de replantear el modelo vigente de representación política, hacia uno que fomente la participación ciudadana y el establecimiento de mecanismos de garantía efectiva de los derechos humanos en un contexto de promoción de una cultura democrática e incluyente.

\section{Abstract:}

This document presents a general reflection on some core issues of the current debate about political participation in a complex society by identifying those political practices that directly affect representation and the representativeness of electoral systems, and in particular, the negative effects of clientelism, given the need to re-think the current political representation model, toward another one that encourages citizen participation and the setting of instruments to achieve an effective guarantee of human rights within a context of promoting a more democratic and evolving culture.

Palabras clave: derecho electoral, representación política, sistema electoral, clientelismo, participación ciudadana.

Descriptors: electoral law, political representation, electoral system, clientelism, citizen participation. 


\section{A manera de introducción}

El propósito de este artículo es destacar algunos aspectos que estimo relevantes dentro de la temática de la Comisión que tengo el honor de presidir, considerando que el diálogo y el debate al que se nos convoca constituyen una magnífica oportunidad para repensar el funcionamiento y la calidad de las instituciones representativas y de otros mecanismos institucionales asociados con los procesos democráticos, destacadamente los sistemas electorales, así como respecto del efecto que, sobre tales procesos, tienen ciertos comportamientos que pueden caracterizarse como "síntomas de deterioro", "patologías" o "comportamientos parasitarios”, como lo señala Ernesto Garzón Valdés (2000: 32-38); entre ellos, el "clientelismo", que afecta, tanto la idea como la práctica de la representación política en Iberoamérica, en un contexto creciente de la desigualdad, exclusión y falta de oportunidades.

La idea principal que someto a debate es que la construcción de ciudadanía pasa necesariamente por el diseño de un modelo de representación política que fomente la participación ciudadana, no sólo en las actividades más características de los sistemas democráticos, como son las elecciones, referendos o consultas populares (ahí donde legalmente son posibles), sino también, y de manera fundamental, en el establecimiento de mecanismos de garantía efectiva de los derechos fundamentales en general, tanto los relacionados directamente con la participación política, como aquellos otros que generan las condiciones sociales, culturales y económicas en un proceso más amplio de promoción de una cultura que fomente el "sentimiento constitucional” - del que hablara, entre otros, Lucas Verdú-que necesariamente pasa por el reconocimiento de los derechos y deberes que le dan sentido y, particularmente, por la construcción de un modelo de sociedad incluyente que garantice no sólo la igualdad de derechos, sino también de oportunidades. 


\section{Participación ciudadana, representación y representatividad}

Los planteamientos en favor de una participación efectiva e incluyente de la ciudadanía en las instituciones representativas y, en general, en el sistema jurídico-político, no sólo inciden en el plano teórico, sino que constituyen reclamos legítimos de la ciudadanía en el orden práctico. De ahí que se afirme y se reitere que la praxis política no puede desvincularse de la teoría a la que se debe, y que reclama eficacia institucional para sus contenidos. Es así que también se alude no sólo a "prácticas políticas" sino a la necesidad de implementar "buenas prácticas". Ello supone, necesariamente, volver al tema de la ética política, a sus posibilidades, alcances y limitaciones, tanto en la práctica como en la teoría.

En cualquier caso, la práctica está inmersa en planteamientos y replanteamientos teóricos que alimentan el debate doctrinario, pero que también inciden en la forma de ejercer el poder político. Como observa Michael Sandel (1998): "Difícilmente podríamos describir nuestra vida política, mucho menos involucrarnos en ella, sin recurrir a un lenguaje cargado de teoría-de derechos y obligaciones, ciudadanía y libertad, democracia y derecho". De ahí que las definiciones construyan modelos institucionales que, a su vez, definen prácticas políticas.

De esta forma, la participación política en la práctica exige definiciones claras de los modelos de representación y la representatividad como factor de legitimación. La efectividad de dicha participación responde, tanto a los principios e ideales que sustentan el modelo democrático, como a la capacidad real que el ejercicio de la representación tiene para cambiar la realidad en beneficio de la ciudadanía, a través de la deliberación, pública y abierta, y de los acuerdos y consensos necesarios.

Una sociedad democrática supone reconocer no sólo el derecho a la participación política sino también la necesidad de establecer las condiciones necesarias para que esta sea posible y resulte efectiva. La participación es, quizá, la primera expectativa de los regímenes democráticos. Desde esta perspectiva, el concepto mismo de ciudadanía se pone sobre la mesa de análisis, tanto desde la perspectiva formal, respecto de la reglamentación del ejercicio de los derechos políticos, 
como de las capacidades reales de las personas para participar políticamente en la comunidad y en la sociedad en la que desarrollan sus vidas, lo que supone pensar en una noción ciudadanía cada vez más incluyente (PNUD, 2004). Superar, de alguna manera esa noción de ciudadanía como privilegio, hacia una noción integrada de la ciudadanía como condición legal y como actividad deseable y posible (Kymlicka y Norman, 1997).

Así, por ejemplo, la 1a. Encuesta Iberoamericana de Juventudes, realizada por la Organización Internacional de Juventud (OIJ), de este año, muestra que los jóvenes en Latinoamérica, España y Portugal esperan que su participación se incremente en el próximo lustro, de forma tal que las instituciones han de propiciar las condiciones que generen la confianza suficiente en la vía institucional como cauce de participación, y proporcionar los espacios que esa mayor participación requiera. De lo contrario, es previsible que esa expectativa y necesidad de participación (como búsqueda de alternativas y como expresión de pertenencia a una comunidad y a una generación política) se canalice por cauces informales o no institucionalizados, en forma de protesta, en calles y plazas, como ha ocurrido en diversos lugares del mundo. El riesgo es que se pierda la confianza en las instituciones y la anomia crezca al punto de deslegitimar al sistema democrático como alternativa de participación eficaz. La representatividad supone en la actualidad no sólo una expectativa ontológica del cuerpo político, sino una directiva deontológica de las condiciones para el mantenimiento del propio sistema democrático.

En este escenario complejo es preciso recordar, como lo expone también la encuesta referida, que los políticos y la policía ocupan los lugares más bajos de confianza entre los jóvenes, quienes representan el núcleo mayoritario de las sociedades en buena parte de los países iberoamericanos, no sólo en el aspecto numérico sino en su valor simbólico desde la perspectiva de la legitimación generacional del régimen político.

Esta circunstancia ha llevado a plantear en el ámbito teórico la idea de la "crisis de la representación política". Al respecto, Roberto Gargarella, en su libro con ese título (2002), muestra que la crisis de las instituciones propias del sistema representativo se debe, en buena medida, a que las mismas fueron diseñadas bajo criterios elitistas, lo que ha tenido como uno de sus varios efectos el marcado aislamiento de los representantes en relación con sus representados. 
Esta revista forma parte del acervo de la Biblioteca Jurídica Virtual del Instituto de Investigaciones Jurídicas de la UNAM

De ahí que sea pertinente y necesario repensar la idea de que la democracia tiene límites en lo concerniente a la participación efectiva de la ciudadanía, como lo hace, por ejemplo, Adam Przeworsky (2010), quien plantea la cuestión de si es posible hacer que la participación política sea más efectiva en cualquier sistema de instituciones representativas en las que el autogobierno se ejerza mediante elecciones.

En mi concepto, la respuesta que se proponga debe pasar por tomar en serio los ideales que han orientado los sistemas políticos en los últimos 200 años, en específico, la libertad, la igualdad y el autogobierno, no desde la perspectiva exclusiva de los derechos y las libertades sino también de las garantías y los deberes.

En lo concerniente a la libertad e igualdad, tiempo atrás, Kelsen observó, en su obra Esencia y valor de la democracia ([1920] 2002), que es característico de la democracia la síntesis de ambos principios, pues, en sus palabras: "Para seguir siendo iguales necesitamos soportar un dominio ajeno", en el entendido de que, como lo afirmó el mismo Kelsen, en una obra posterior: "Políticamente libre es el individuo que se encuentra sujeto a un ordenamiento jurídico en cuya creación participa" ([1949] 1983), pero no cualquier dominio, y en modo alguno, un dominio absoluto, ya que también, como advierte Philip Pettit (1999), la libertad como no-dominación constituye un valioso ideal político.

Esta interrelación entre igualdad y libertad se manifiesta también en la correlación necesaria entre derechos y deberes, entendidos los primeros como parte de un proceso de institucionalización del poder y de su legitimación, y los segundos como parte indispensable de cualquier noción de solidaridad y responsabilidad social; incluyendo aquí también lo que Garzón Valdés llama los deberes positivos generales (2000: 127 y ss.), que responden, de manera más general a las necesidades humanas del mundo contemporáneo que no sólo requieren discursos teóricos, sino, y con más urgencia, medidas específicas que incidan y modifiquen la realidad, a través de la praxis mediante la cual se autoconstruye.

En lo referente al autogobierno, coincido en que las elecciones periódicas constituyen la institución fundamental del mismo (Przeworsky, 2010); no obstante, la idea de autogobierno está sujeta a tensiones evidentes, en atención al reconocimiento de la complejidad y pluralidad de las sociedades contemporáneas.

Ello nos lleva a la pregunta de cuáles son las condiciones que debe satisfacer un sistema de toma de decisiones colectivas para reflejar 
adecuadamente las preferencias individuales, haciendo lo más libre posible a la mayor cantidad de personas. Sobre este tema se han ofrecido varias respuestas y muchas cuestiones siguen abiertas.

En primer término, se requiere una mayor precisión conceptual, lo que supone no equiparar "régimen democrático" con "sistema representativo", ya que, como ha señalado Bovero, un Estado representativo no es necesariamente un Estado democrático (2002). El propio Bovero aporta algunas directivas, al destacar que los elegidos en un parlamento representan a los ciudadanos electores en forma democrática, no solamente en la medida en que son designados por éstos para sustituirlos en las fases conclusivas del proceso decisional, sino en la medida en que el parlamento, en su conjunto y en sus varios componentes, refleja las diversas tendencias y orientaciones políticas existentes en el país, considerando éste de manera global, sin exclusiones, y en sus respectivas proporciones.

Tal perspectiva destaca, cuando menos, dos temas cruciales: el primero, el significado de la propia idea de representación, y, el segundo, el de la necesaria proporcionalidad de los sistemas electorales, en atención a las sociedades plurales y a la multiculturalidad presente en todas ellas.

En ese sentido, en mi concepto, una discusión de tales temas ha de incluir, entre otros, los siguientes aspectos:

- La necesidad, importancia y legitimidad de proteger jurisdiccionalmente de forma efectiva los derechos humanos de participación política y la celebración de elecciones periódicas y auténticas en las que se cumpla con determinados principios o valores para tenerlas como democráticas.

- Incluir figuras procesales que fomenten la apertura de los procesos interpretativos, tales como el amicus curiae, y de las acciones para la defensa de intereses difusos y colectivos.

- La tutela de la democracia en el interior de los partidos políticos, a fin de desactivar sus tendencias oligárquicas, mediante la protección efectiva de los derechos de sus afiliados o militantes, fortaleciendo, al mismo tiempo, el principio de autoorganización de los partidos políticos y el principio de libre participación política.

- El fortalecimiento de la democracia deliberativa.

- El reconocimiento del derecho de los pueblos y comunidades indígenas a la autodeterminación, lo que incluye el derecho a la 
consulta previa respecto de medidas que los afecten, así como las necesarias garantías para su ejercicio.

- La garantía de participación política de las mujeres en condiciones de igualdad.

- La revisión de las limitaciones existentes a los derechos políticos, entre otras, las relativas a las personas privadas de su libertad por investigación o condena judicial.

- El reconocimiento y adecuada reglamentación de las candidaturas independientes como alternativas de participación ciudadana.

- El establecimiento de instituciones como el derecho de revocación de mandato de los representantes u otros mecanismos para hacer responsables a los representantes frente a los representados, y

- Repensar los modelos de comunicación política y del uso de las tecnologías de la información y comunicación para fomentar la participación de la ciudadanía y la rendición de cuentas de representantes en el marco de una democracia, cada vez más "digital".

De esta forma, de un adecuado diseño institucional depende el grado de representatividad geográfica, étnica, poblacional e ideológica del sistema político y de éste depende, en buena medida, el grado de legitimación del mismo; asimismo, del grado de legitimidad y de la calidad de la representación, depende el nivel de participación ciudadana y de la intensidad de ésta la conformación de un consenso constitucional en el cual la ciudadanía opere, no sólo como puerta de acceso a la participación y representación política, sino también como herramienta para el control de los poderes públicos y para el ejercicio de los derechos y libertades fundamentales.

\section{Sistemas electorales}

Es bien sabido que los sistemas electorales constituyen el mecanismo jurídico mediante el cual se definen los elementos que permiten traducir los votos de los electores en escaños parlamentarios, para el efecto de integrar los órganos de gobierno o de la asamblea representativa. El debate entre sistemas electorales se inscribe también en la discusión 
Esta revista forma parte del acervo de la Biblioteca Jurídica Virtual del Instituto de Investigaciones Jurídicas de la UNAM

sobre las ventajas y desventajas existentes entre los diferentes modelos de sistemas políticos: parlamentario, presidencial o mixto. ${ }^{1}$

La determinación del sistema electoral es una decisión institucional fundamental en toda sociedad democrática en la que convergen intereses políticos y expectativas ciudadanas, y forman parte esencial de la cadena que conecta las preferencias de los ciudadanos con las decisiones políticas que toman los órganos representativos y de gobierno (Gallagher y Mitchell, 2005).

Dada su importancia, son muchos los estudios y planteamientos sobre los elementos, características, tipologías y contextos en el diseño institucional de los sistemas electorales. Para efecto de esta presentación y sólo para ilustrar la complejidad del tema, me remito a lo expresado por Dieter Nohlen, en el sentido de que, para la selección de un adecuado sistema electoral, se tienen que tomar en consideración las siguientes "variables contextuales": $i$ ) estructura social; $i$ i) cantidad y profundidad de líneas de conflicto; iii) grado de fragmentación del sistema de partidos; iv) grado de institucionalización del sistema de partidos; $v$ ) modelo de interacción entre los partidos; vi) dispersión de los votantes, y vii) comportamiento del electorado (Nohlen, 2008).

Como resulta evidente, no existe un modelo ideal y, en el transcurso de los años, el funcionamiento real de los sistemas electorales ha arrojado como evidencia que cada uno posee ventajas y desventajas. Son conocidos los debates sobre los diferentes sistemas electorales y existe una amplia bibliografía sobre el tema, sin que exista un consenso acerca de cuál es el mejor sistema electoral, ya sea de mayoría, de representación proporcional o mixto, en sus diferentes variables y formas de votación, considerando sus efectos no sólo en la representación, a través del "efecto mecánico" de distribución de los votos, sino también en el "efecto psicológico" que se refleja en un mayor o menor grado de participación política. ${ }^{2}$

Aquí, me limito a señalar algunos aspectos que han sido destacados como parámetros a considerar en el diseño de los sistemas electorales y en cualquier modelo de ajuste que se pretenda para garantizar no sólo su eficacia sino también la confianza y accesibilidad en la elección misma: el grado de gobernabilidad efectiva; la adecuada

\footnotetext{
1 En general véase: Aragón, 1998; Valadés y Serna, 2000, y Ellis, Orozco y Zovatto, 2009.

2 En general, véase, Reynolds et al., 2006.
} 
Esta revista forma parte del acervo de la Biblioteca Jurídica Virtual del Instituto de Investigaciones Jurídicas de la UNAM

representación de las mujeres y la efectiva representación de grupos minoritarios, pueblos indígenas y personas en situaciones específicas (migrantes, discapacitados, extranjeros residentes, etcétera); sus efectos en el sistema de partidos; la apertura a las candidaturas independientes; el grado de representatividad geográfica, ideológica, política y poblacional y, en última instancia, el grado de legitimación del sistema representativo.

Dentro de los temas a debate, destacan también los efectos de los sistemas de votación, sus ventajas o desventajas, en el diseño institucional, entre ellos, el voto alternativo, la doble ronda, el voto transferible o no transferible, así como las modalidades del voto obligatorio, del voto en ausencia, o del voto desde el extranjero. Cuestiones que, necesariamente, trascienden a otros aspectos relevantes de naturaleza financiera o administrativa, y que inciden en la legitimación del régimen democrático, como la transparencia y la rendición de cuentas; aunado al hecho de que el diseño del sistema electoral, cualquiera que sea su modalidad, incide en el conjunto del sistema democrático y de su estructura institucional, así como en otras manifestaciones del ejercicio del poder político, tanto al interior de los partidos como de los grupos parlamentarios.

Asimismo, es importante incorporar el tema de la accesibilidad y la no discriminación, tanto en la representación como en la participación. Esto es, impulsar condiciones de equidad y promover candidaturas representativas de personas, grupos minoritarios o en situación de desventaja, y garantizar también medidas adecuadas para que estas personas puedan ejercer sus derechos políticos de votar y ser votado, incluso considerando los modelos de papeletas electorales, lugares y fechas de votación.

En cualquier caso, resulta decisivo elegir el sistema electoral en función del contexto político-social de cada país, ya que éste impacta en diversas variables: $i$ ) el sistema de partidos políticos; ii) la naturaleza del gobierno, esto es, si será de coalición, de mayoría o de partido único; iii) el tipo de decisiones que enfrenta el electorado en las elecciones; iv) la capacidad del electorado para exigir rendición de cuentas a sus representantes; $v$ ) el grado de democracia y la cohesión dentro de los partidos políticos; vi) la calidad del gobierno, y vii) la calidad de vida los gobernados (Gallagher y Mitchell, 2005).

Al respecto, cabe tener presente que la Corte Interamericana de Derechos Humanos ha precisado que, más allá de ciertas características del proceso electoral (universal, igual, secreto, que refleje la libre ex- 
presión de la voluntad popular), la Convención Americana de Derechos Humanos "no establece una modalidad específica o un sistema electoral particular mediante el cual los derechos a votar y ser elegido deben ser ejercidos". La Convención - agrega la Corte Interamericana- se limita a establecer determinados estándares dentro de los cuales los Estados legítimamente pueden y deben regular los derechos políticos, siempre y cuando dicha reglamentación cumpla con los requisitos de legalidad, esté dirigida a cumplir con una finalidad legítima, sea necesaria y proporcional; esto es, sea razonable de acuerdo con los principios de la democracia representativa (caso Castañeda Gutman vs. México).

Como lo advierte el Nuevo Manual de IDEA Internacional, "la construcción de instituciones políticas no sólo es una tarea clave para las nuevas democracias sino también para las establecidas que están buscando modificar sus sistemas para reflejar de mejor forma las nuevas realidades políticas", considerando además la importancia del contexto dentro del cual se selecciona un determinado sistema electoral y el interés general de "adoptar instituciones que sean suficientemente fuertes para promover una democracia estable, pero suficientemente flexibles para ajustarse a circunstancias cambiantes" (2006: 2).

Finalmente, reitero la importancia del contexto en la operación de los sistemas electorales y la incidencia que éstos tienen, conjuntamente con otros mecanismos de participación directa, como la consulta popular, la iniciativa ciudadana o el referéndum, en el conjunto del sistema político y del régimen democrático. En última instancia, si bien el modelo de sistema electoral es una decisión política que se traduce en fórmulas jurídicas y diseños institucionales, su eficacia y permanencia dependen, no sólo de su propio diseño, sino también del contexto cultural y político en el que operan.

\section{El clientelismo o la instrumentalización de la participación política}

El "clientelismo" es una práctica social ampliamente conocida y comentada en el escenario político-electoral, incorporada formal o informalmente al sistema político. Entendido tradicionalmente como el intercambio de bienes y servicios, favores o tratos privilegiados, por 
Esta revista forma parte del acervo de la Biblioteca Jurídica Virtual del Instituto de Investigaciones Jurídicas de la UNAM

apoyo político y, en específico, por votos en una elección, se reconoce también como un fenómeno social y jurídico mucho más complejo, multidimensional, que forma parte de un imaginario colectivo y simbólico, que genera relaciones sociales y, en muchas ocasiones, vínculos de interés, reciprocidad, lealtad, confianza y subordinación, con cierta estabilidad y permanencia; "cadenas de clientelismo" que si bien representan formas de organización y acción política, sumadas a otras prácticas, como la corrupción y el patronazgo - que inciden directamente sobre la distribución de bienes y cargos públicos- generan relaciones marcadamente desiguales o asimétricas que constituyen comportamientos parasitarios que poco a poco erosionan la legitimidad de los procesos y gobiernos democráticos. ${ }^{3}$

En el ámbito electoral, el clientelismo implica el reparto de favores, bienes materiales, servicios o dinero a cambio de votos y/o apoyo político, y se manifiesta tanto de manera formal como informal; de manera formal a través de la distribución de la denominada "propaganda utilitaria”, ahí donde, como en México, está permitida, ${ }^{4}$ y, de manera informal, a través de redes de intercambio que suelen estar marcadas por la desigualdad entre los sujetos implicados, y tienden a lucrar con las necesidades y la pobreza de las personas menos favorecidas por el sistema económico-social, con independencia de que resulten mecanismos asistencialistas que puedan generar algún tipo de "alivio" momentáneo o expectativa futura de solución a su problemática.

Desde esta perspectiva, el clientelismo supone la instrumentalización de la participación política a partir de la permanencia de un estado de exclusión y dependencia. Una práctica que si bien, en general, es intermitente, limitada a ciertos periodos - como los procesos electorales- opera sobre y reclama para sí condiciones de desigualdad permanentes, con independencia de que las prácticas clientelares supongan intercambios de votos por favores de manera informada y calculada, como parte de una estrategia no sólo de los partidos políticos

3 En general, entre otros: Auyero, 2002; Corrochano, 2002; Espinal, 2003; González Alcantud, 1997; Gordín, 2006; Schröter, 2010, y Szwarcberg, 2012.

${ }_{4}$ Ejemplos de denuncias de estas prácticas y de su análisis en sede judicial pueden verse en algunos precedentes de la Sala Superior del Tribunal Electoral del Poder Judicial de la Federación de México, por ejemplo en las sentencias relativas al juicio de inconformidad SUP-JIN-359/2012, sobre la pretensión de nulidad de la elección presidencial de 2012 y a los recursos de apelación SUP-RAP-8/2013 y SUP-RAP-14/2013 acumulados (Caso sobre el concurso Círculo Verde, campaña PVEM 2012), en: http://www.te.gob-mx. 
Esta revista forma parte del acervo de la Biblioteca Jurídica Virtual del Instituto de Investigaciones Jurídicas de la UNAM

sino también de los ciudadanos y de organizaciones populares, pues tales relaciones, generalmente ocultas o negadas, suponen patrones disfuncionales de la participación política, al centrarse en la resolución de problemas inmediatos y particularizados sobre la asunción de dudosas lealtades, fomentando la desmovilización, la erosión de vínculos de solidaridad y la desintegración paulatina de la relación entre representado y representante, cuya finalidad supone atender problemas que involucran intereses sociales de mayor calado.

En cualquier caso, esas relaciones asimétricas generan presión sobre los electores, lo mismo que estímulos para su participación. De esta forma, la instrumentalización del voto a cambio de la entrega de bienes, servicios o promesas de apoyos o favores de cualquier índole (política, económica, administrativa, judicial, fiscal, etcétera) hace más difícil conocer la "verdadera" motivación del elector al momento de votar y, con ello, escrutar las razones subyacentes a su participación en los procesos político-electorales, con independencia de que sea una práctica ilícita o no.

Sin duda, una cuestión fundamental al momento de valorar la incidencia de estas prácticas en el sistema político es su grado de institucionalización formal o informal, a partir no sólo de su concurrencia en los procesos electorales, sino de su incidencia en los resultados; así como la adecuada identificación de las condiciones que nutren al clientelismo, como es su grado de legitimidad en el sistema de valores de una comunidad, la pobreza, la desigualdad, la exclusión económica, social y política, la corrupción, el patronazgo, la falta de transparencia y rendición de cuentas, el nivel de acceso a la información relevante, la capacidad de autoorganización de la comunidad frente a los grupos de presión y de interés político, la falta de recursos efectivos para hacer valer derechos fundamentales - en particular aquellos de naturaleza económica, social y cultural-y, en definitiva, el nivel de la ética pública en la distribución de los bienes y servicios, también públicos, a partir de un criterio universal e incluyente y no de uno particularista, personal y excluyente.

Resurge aquí también la relación entre derechos y deberes, y entre la estrategia electoral y la ética pública. Ahí donde la "sustentabilidad política" depende de los lazos clientelares, del patronazgo y de la desigualdad estructural, el clientelismo arraiga y, junto con otras prácticas, hace más difícil la adopción de medidas efectivas para erradicarlas o mantenerlas en márgenes razonables (Gordin, 2006). Lo mismo 
sucede ahí donde el clientelismo es un mecanismo no sólo de cooptación y presión del voto, sino también de "alivio" (real o aparente) de situaciones de inseguridad económica, insatisfacción o exclusión social y política. En cuanto tal, el clientelismo constituye un rasgo del sistema político que no debe sobreestimarse ni subestimarse en sus consecuencias y efectos.

Romper las redes clientelares y el patronazgo supone la construcción de nuevas redes sociales, de nuevas solidaridades y de transparencia en las relaciones entre las autoridades públicas y la sociedad. De esta forma, la participación como ejercicio de autonomía, como integración a la comunidad política y como fuente de confianza individual y solidaridad colectiva puede ser una forma de reducir los efectos parasitarios de las redes clientelares.

\section{Comentario final}

La presente reflexión pretende ser una introducción al debate sobre los temas que ocupan esta Comisión sobre la base de entender la participación ciudadana como un elemento fundamental en el proceso de institucionalización de prácticas políticas que inciden directamente en la representación, en la representatividad de los sistemas electorales y en prácticas como el clientelismo y el patronazgo.

La instrumentalización de la participación ciudadana a través de prácticas clientelares erosiona la idea de solidaridad e interés común como base de la convivencia social. Es necesario replantear el modelo de participación basado en el clientelismo, a la luz de un modelo de representación basado en la participación ciudadana libre e informada, pero también generar alternativas de participación que modifiquen modelos de creencias compartidas que legitiman la estrategia clientelar en los procesos políticos.

Ello requiere no sólo de una perspectiva de análisis adecuada a la complejidad y muldimensionalidad del fenómeno, sino también la adopción de medidas en otros terrenos que de manera directa o indirecta inciden en el grado y la calidad de la representación, entre ellas aquellas medidas encaminadas a perfeccionar el sistema de fiscalización de los recursos de los partidos; brindar mayor capacitación y educación; garantizar la existencia de una pluralidad de medios de 
información; sancionar la compra y coacción del voto; promover y garantizar el uso transparente de los recursos públicos, incrementar la institucionalización de servicio civil de carrera y la profesionalización de la administración; generar mayores oportunidades de empleo y una distribución más equitativa de los servicios públicos; así como establecer recursos judiciales efectivos para la protección de los derechos humanos tanto civiles y políticos, como económicos, sociales y culturales.

La construcción de una ciudadanía plena pasa por configurar un modelo de la participación y representación que procure trasladar las directivas legales en modelos de convivencia, y éstos en escenarios de legitimación del sistema político. Un modelo que no se limite a invocar aspectos formales de la representación sino que incida en el ejercicio cotidiano de los derechos y en el cumplimiento espontáneo de los deberes, en la concurrencia de ambos no sólo en la ley sino también en la plaza, como espacio de convivencia y como escenario de transformación.

\section{Bibliografía}

Aragón, Manuel, 1998, "Sistema parlamentario, sistema presidencialista y dinámica entre los poderes del Estado. Análisis comparado", en Pau I Vall, Francesc (coord.), Parlamento y Control de Gobierno. $V$ Jornadas de la Asociación Española de Letrados Parlamentarios, España, Aranzadi.

Auyero, Javier, 2002, "Clientelismo político en Argentina: doble vida y negación colectiva”, Perfiles Latinoamericanos. Revista de la Facultad Latinoamericana de Ciencias Sociales, Sede México, núm. 20, junio.

Bovero, Michelangelo, 2002, Una gramática de la democracia. Contra el gobierno de los peores, trad. de Lorenzo Córdova, Madrid, Trota.

Carrochano, David H., 2002, "El clientelismo posmoderno", Perfiles Latinoamericanos. Revista de la Facultad Latinoamericana de Ciencias Sociales, Sede México, núm. 20, junio.

Corte IDH, Caso Castañeda Gutman vs. México, excepciones preliminares, fondo, reparaciones y costas, sentencia del 6 de agosto de 2008, serie C, núm. 184. 
Ellis, Andrew et al. (coords.), 2009, Cómo hacer que funcione el sistema presidencial. Making Presidentialism work, México, UNAM-IIJCAPEL-IDEA International.

EsPinal, Rosario, "Clientelismo", Diccionario Electoral, México, CAPEL-IFE-TEPJF-UNAM, 2003.

Gallagher, M. y Mitchell, P. (coord.), 2008, The Politics of Electoral Systems, Oxford University Press.

Gargarella, Roberto, 2002, Crisis de la representación política, México, Fontamara.

Garzón VAldÉs, Ernesto, 2000, Instituciones suicidas. Estudios de ética y política, México, UNAM-Paidós.

González Alcantud, José Antonio, 1997, El clientelismo político: Perspectiva socioantropológica, Rubí (Barcelona), Anthropos Editorial.

Instituto Federal Electoral, 2011., "Elecciones presidenciales y legislativas en 18 países de América Latina”, Estudios Electorales en Perspectiva Internacional Comparada, México, Instituto Federal Electoral

Kelsen, Hans, 2002, Esencia y valor de la democracia, trad. de Luis Legaz Lacambra, Granada, Comares [1920].

__—, 1983, Teoría general del derecho y del Estado, trad. de Eduardo García Máynez, México, UNAM, [1949].

Kymlicka, Will y Norman, Wayne, 1997, "El retorno del ciudadano. Una revisión de la producción reciente en teoría de la ciudadanía", La Política. Revista de estudios sobre el Estado y la sociedad, núm. 3, octubre, pp. 5-39.

Lijphart, A. y Grofman, B. (coord.) , 1984, Choosing an Electoral System, Issues and Alternatives, Praeger.

Lijphart, A., 2000, Modelos de democracia, Formas de gobierno y resultados en treinta y seis países, Ariel.

Nohlen, D., 2008, Sistemas electorales en su contexto. Suprema Corte de Justicia de la Nación, UNAM, Instituto de Investigaciones Jurídicas y Benemérita Universidad Autónoma de Puebla.

Nohlen, D. et al. (comp.), 1998, Tratado de derecho electoral comparado de América Latina, Instituto Interamericano de Derechos Humanos-Universidad de Heidelberg Instituto de Ciencia PolíticaTribunal Electoral del Poder Judicial de la Federación-Instituto Federal Electoral-Fondo de Cultura Económica. 
Norris, P., 1997, “Choosing Electoral Systems: Proportional, Majoritarian and Mixed Systems", International Political Science Review, vol. 18 (3), julio.

Organización Internacional de Juventud, El Futuro ya llegó. $1^{a}$ Encuesta Iberoamericana de Juventudes (Informe Ejecutivo), México, OIJ-BID-CAF-UNAM-PNUD-CEPAL, 2013.

Petit, Philipp, Republicanismo. Una teoría sobre la libertad y el gobierno, trad. de Tony Domenech, Buenos Aires, Paidós, 1999.

PNUD, La Democracia en América Latina. Hacia una democracia de ciudadanas y ciudadanos, PNUD, Perú, 2004.

PrZeworsky, Adam, Qué esperar de la democracia. Límites y posibilidades del autogobierno, trad. de Stella Mastrangelo, Argentina, Siglo XXI Editores, 2010.

SChedLer, Andreas, 2004, "El voto es nuestro: ¿cómo los ciudadanos mexicanos perciben el clientelismo electoral?", Revista Mexicana de Sociología, 66, núm. 1, enero-marzo.

SCHröter, Barbara, 2010, "Clientelismo político: ¿existe el fantasma y cómo se viste?”, Revista Mexicana de Sociología, 72, núm. 1, eneromarzo.

Szwarcberg, Mariela, 2012, “Actos partidarios y clientelismo político en América Latina", Nueva Sociedad, núm. 240, julio-agosto.

Reynolds, Andrew et al. (coords.), Diseño de sistemas electorales. Nuevo manual de IDEA Internacional. México, IFE-IDEA Internacional-TEPJF, 2006.

VAladés, Diego y Serna, José María (coords.), 2000, El gobierno en América Latina ¿Presidencialismo o parlamentarismo?, México, UNAM, Instituto de Investigaciones Jurídicas. 